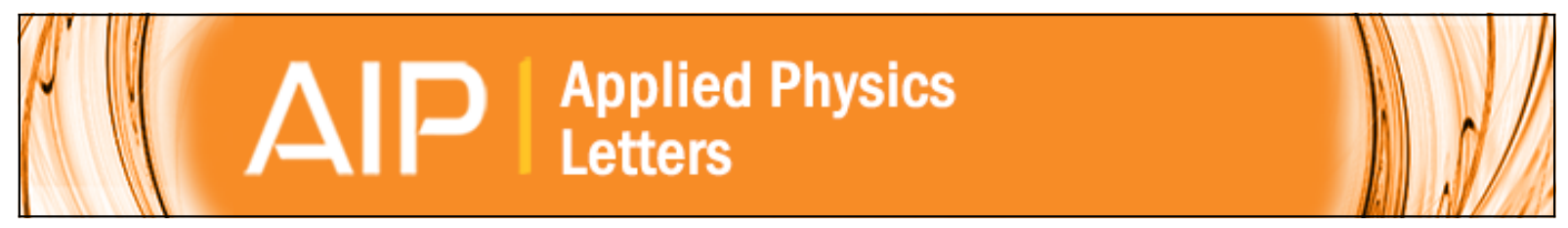

Local magnetometry at high fields and low temperatures using InAs Hall sensors

E. Pugel, E. Shung, T. F. Rosenbaum, and S. P. Watkins

Citation: Applied Physics Letters 71, 2205 (1997); doi: 10.1063/1.120443

View online: http://dx.doi.org/10.1063/1.120443

View Table of Contents: http://scitation.aip.org/content/aip/journal/apl/71/15?ver=pdfcov

Published by the AIP Publishing

Articles you may be interested in

Large voltage modulation in magnetic field sensors from two-dimensional arrays of $\mathrm{Y}-\mathrm{Ba}-\mathrm{Cu}-\mathrm{O}$ nano Josephson junctions

Appl. Phys. Lett. 104, 062601 (2014); 10.1063/1.4865216

Three-axis Hall transducer based on semiconductor microtubes

Appl. Phys. Lett. 103, 173513 (2013); 10.1063/1.4826680

Mobile high-temperature superconductor dc superconducting quantum interference device cooled by a pulsetube cooler

Rev. Sci. Instrum. 76, 074704 (2005); 10.1063/1.1946927

InAs/Al 0.2 Ga 0.8 Sb quantum well Hall sensors with improved temperature stability

Rev. Sci. Instrum. 70, 2715 (1999); 10.1063/1.1149834

Measurement of the magnetic induction vector in superconductors using a double-layer Hall sensor array Appl. Phys. Lett. 72, 2891 (1998); 10.1063/1.121450

\title{
A|P| Journal of
}

Journal of Applied Physics is pleased to announce André Anders as its new Editor-in-Chief 


\title{
Local magnetometry at high fields and low temperatures using InAs Hall sensors
}

\author{
E. Pugel, E. Shung, and T. F. Rosenbaum ${ }^{\mathrm{a})}$ \\ The James Franck Institute and Department of Physics, The University of Chicago, Chicago, Illinois 60637 \\ S. P. Watkins \\ Department of Physics, Simon Fraser University, Burnaby, British Columbia V5A 1S6, Canada
}

(Received 13 June 1997; accepted for publication 6 August 1997)

\begin{abstract}
We characterize the temperature $(0.3 \leqslant T \leqslant 300 \mathrm{~K})$, magnetic field $(0 \leqslant H \leqslant 80 \mathrm{kOe})$, and thickness $(0.1,0.5$, and $2.5 \mu \mathrm{m})$ dependence of the Hall response of high purity InAs epilayers grown using metalorganic chemical vapor deposition. The high sensitivity, linearity, and temperature independence of the response make them attractive for local Hall probe magnetometry, and uniquely qualified for high field applications below liquid helium temperatures. As a stringent test of performance, we use a six element micron-sized array to monitor the internal field gradient during vortex avalanches at milliKelvin temperatures in a single crystal of $\mathrm{YBa}_{2} \mathrm{Cu}_{3} \mathrm{O}_{7-\delta}$. (C) 1997 American Institute of Physics. [S0003-6951(97)00941-8]
\end{abstract}

The response of electronic and spin systems to an external magnetic field provides essential information about the microscopic characteristics of a material. In a conventional measurement, the entire sample is probed at once, providing information about the global magnetic behavior. In investigations of systems like spin glasses, where there is no longrange magnetic order, or type II superconductors, where spatially separated, quantized units of magnetic flux penetrate the crystal or film, it is necessary to ascertain the local magnetic state.

The need for high sensitivity magnetization data with a spatial resolution on a length scale of microns or below has become particularly acute with the advent of high $T_{c}$ superconductivity. The easy flow of vortices in the mixed state has limited practical applications of the cuprate superconductors where high-field magnets and high-current capacity wires would be useful, focusing efforts on understanding the mechanisms of vortex dynamics and pinning in these extremely type II materials. A large variety of local probes have been employed, including Bitter decoration, microscopic superconducting quantum interference device (SQUID) loops, Hall bar magnetometers, and magnetooptical imaging. Of these, Hall probe magnetometry is the only method which works at magnetic fields $H \geqslant 1$ kOe.

In operation, one presses a Hall bar against the sample of interest and solves for the magnetization via a comparison of the voltage induced by an external field and the internal magnetic field threading the sample/probe combination. Accurate and sensitive magnetization measurements require a large, linear response up to large $H$, weak dependence on temperature, and reproducibility after thermal cycling. The field from a local magnetic object such as a vortex quickly bends away from the surface perpendicular, leading to the additional requirement that the Hall bar be no thicker than the scale of spatial variations in the measured field distribution. Moreover, the Hall voltage increases linearly with decreasing thickness. Hence, thin film devices are preferable if materials of sufficiently high quality are available. When grown as a

a)Electronic mail: tfr@rainbow.uchicago.edu thin film, the Hall probe then can be patterned as a multielement array on small length scales ${ }^{1}$ using standard lithographic techniques.

The recent development ${ }^{2}$ of high purity InAs epilayers with specular surfaces using metalorganic chemical vapor deposition (MOCVD) techniques has led us to explore the high magnetic field behavior of these films and to exploit them as the active elements of Hall probe arrays. In this letter, we describe how high fields actually reduce the pronounced temperature dependence of the Hall response observed in the low field limit, apparently by suppressing the competition between bulk and surface conduction. A response up to $20 \mathrm{~m} \Omega / \mathrm{G}$ from $\mathrm{mK}$ to room temperature is possible for micron-sized sensors with no saturation up to at least $80 \mathrm{kOe}$. As a test of the sensitivity and reproducibility of these InAs magnetometers, we map out the spatial variation of the vortex density in single crystals of $\mathrm{YBa}_{2} \mathrm{Cu}_{3} \mathrm{O}_{7-\delta}$ cooled to milliKelvin temperatures. The absence of quantum Hall steps at low $T$ makes these sensors uniquely qualified for high field measurements below liquid helium temperatures.

Narrow gap semiconductors like InSb and InAs derive their large Hall effect from a small effective mass which permits metallic conduction at an unusually low carrier density. ${ }^{3}$ The potential of InAs for local magnetometry was recognized nearly 30 years ago by Simpkins ${ }^{4}$ and single crystals of InAs have been used by Konczykowski, Holtzberg, and Lejay ${ }^{5}$ and by Koziol and Franse ${ }^{6}$ for global magnetization measurements of superconductors. True local measurements down to low temperature have not been reported, although the high sensitivity, linearity, and temperature independence of thin InAs epilayers make them attractive for many applications.

We compare in Fig. 1 the Hall resistance as a function of applied field at $\mathrm{mK}$ temperatures for $0.1-\mu \mathrm{m}$-thick polycrystalline $\mathrm{Bi}$ on a sapphire substrate, $0.1-\mu \mathrm{m}$-thick InAs on a GaAs substrate, and a GaAs two-dimensional electron gas heterojunction. ${ }^{7}$ Bismuth is a semimetal with low carrier density commonly used as a Hall probe. Its response is more than an order of magnitude smaller than InAs, with a ten- 


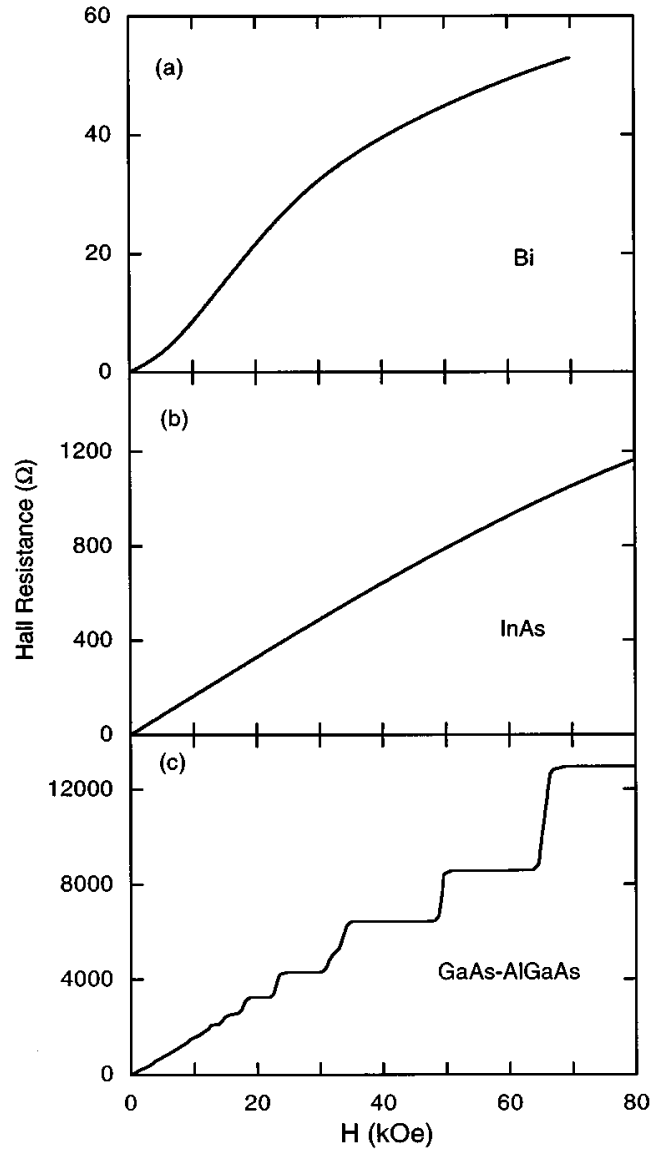

FIG. 1. Comparison of the Hall response as a function of magnetic field $H$ at $T=0.3 \mathrm{~K}$ for three magnetometer materials. Note the change of vertical scale between panels. The GaAs-AlGaAs data is from Ref. 7.

dency towards saturation for $H>30 \mathrm{kOe}$. Furthermore, the Hall constant of $\mathrm{Bi}$ depends strongly on temperature and $\mathrm{Bi}$ films are notoriously sensitive to cracking under thermal cycling, often leading to performance degradation. By comparison, the Hall resistance of InAs remains essentially linear in $H$ up to high field and we find $<0.05 \%$ change in $R(H, T)$ after numerous thermal cycles between room and liquid helium temperatures. The formidable response of the GaAs$\mathrm{AlGaAs}$ heterojunction makes it an excellent material choice at liquid nitrogen temperatures, ${ }^{8}$ but as is clear in Fig. 1(c), the development of quantum Hall plateaus excludes low temperature applications over a sizable field range.

Not only does the Hall resistance of InAs depend linearly on $H$, but its slope changes less than $10 \%$ over 3 decades in temperatures $(0.3<T<300 \mathrm{~K})$. This permits highly accurate magnetization measurements as a function of both field and temperature. We demonstrate the weak temperature dependence in Fig. 2, comparing $R(H, T=5 \mathrm{~K})$ and $R(H, T$ $=300 \mathrm{~K}$ ) for a $0.1-\mu \mathrm{m}$-thick InAs film. In fact, the response changes by only $2 \%$ over the lowest two decades in $T$ (Fig. 2 inset).

The dependence of the Hall response on the thickness of the InAs epilayers is more complicated and results from the competition between conduction in a surface accumulation layer, which contributes most of the free carriers, and a high purity bulk layer which dominates transport at intermediate temperatures. ${ }^{2,9}$ As the $0.1 \mu \mathrm{m}$ InAs prelayer is thickened,

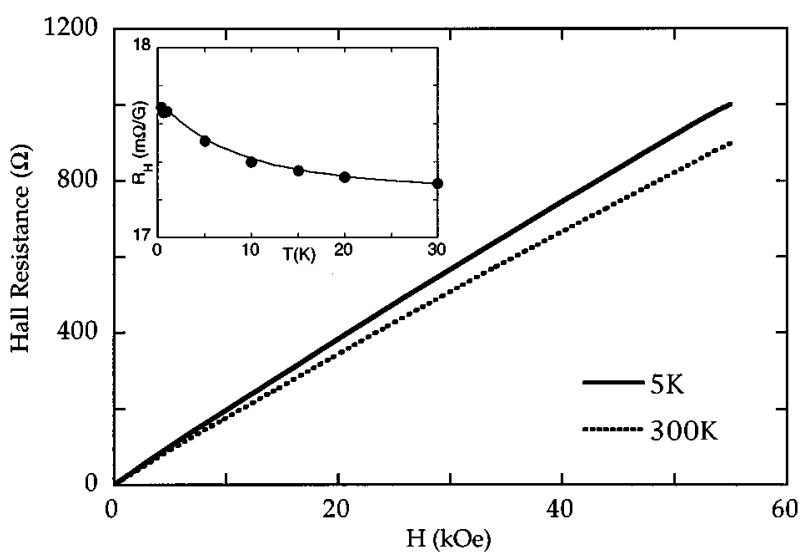

FIG. 2. The Hall resistance vs magnetic field $H$ at room and liquid helium temperatures for a $0.1-\mu \mathrm{m}$-thick epilayer of InAs. The essentially linear response is only weakly temperature dependent. (Inset) The Hall coefficient changes $2 \%$ between 0.3 and $30 \mathrm{~K}$, making device calibration simple.

the Hall mobility increases, but the effective sheet concentration decreases as more mobile, but sparser bulk carriers join the surface layer conduction. The low field response is enhanced at the cost of significant temperature dependence. We show in Fig. 3, however, that the temperature dependence in thicker films (e.g., $0.5 \mu \mathrm{m}$ ) is effectively quenched for $H>20 \mathrm{kOe}$, reverting to the $0.1 \mu \mathrm{m}$ prelayer-type behavior where surface and GaAs interface scattering dominate.

The choice of InAs film thickness then depends on the experiment at hand. If precise temperature scans are required at fields below $20 \mathrm{kOe}$ or if the field lines of local objects like vortices in superconductors or domain walls in ferromagnets need to be imaged, then the $0.1-\mu \mathrm{m}$-thick InAs sensor is the appropriate choice. For experiments where the absolute size of the Hall signal is pivotal, then thicker epilayers may be more suitable. Such devices can have higher peak sensitivity, but with a significant temperature dependent response. We plot in Fig. 4 performance contours in the $H-T$ plane for a $2.5-\mu \mathrm{m}$-thick, high-mobility ${ }^{2}$ InAs device. The bulk conduction provides up to a factor of three enhancement $(60 \mathrm{~m} \Omega / \mathrm{G}$ at $T=77 \mathrm{~K}$ ), but only for $H<20 \mathrm{kOe}$ and 10

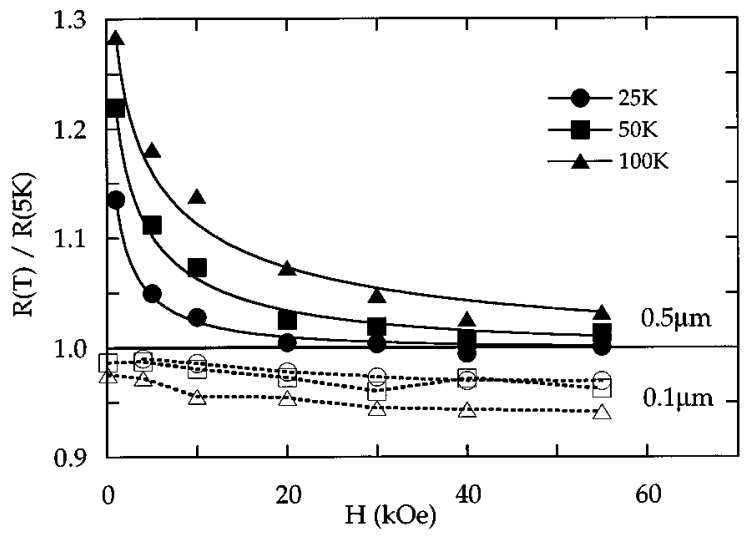

FIG. 3. The Hall resistance at $T=25,50$, and $100 \mathrm{~K}$ normalized to its $T$ $=5 \mathrm{~K}$ value vs magnetic field for $0.1-$ and $0.5-\mu \mathrm{m}$-thick InAs films. The bulk carrier conduction of the thicker film is frozen out for $H>20 \mathrm{kOe}$, reducing the temperature dependence. 


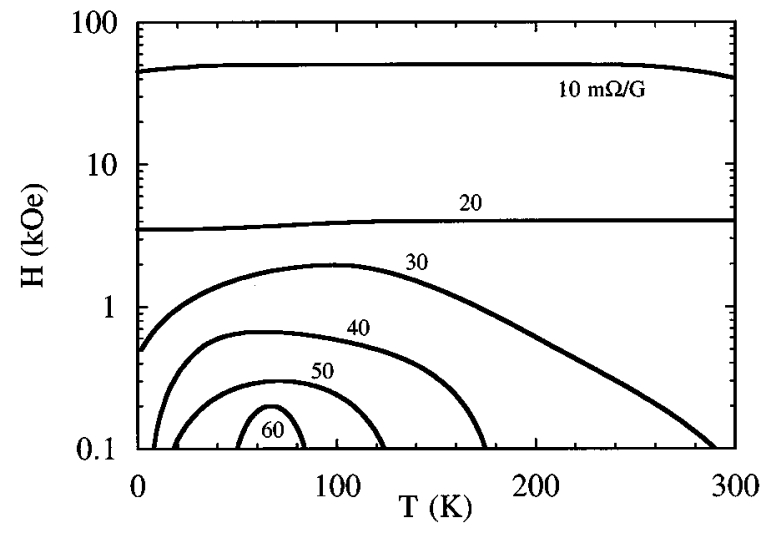

FIG. 4. Contour plot in the $H-T$ plane of the Hall coefficient in $\mathrm{m} \Omega / \mathrm{G}$ for a $2.5-\mu \mathrm{m}$-thick InAs film. Enhanced device sensitivity comes at the cost of significant field and temperature dependence.

$<T<200 \mathrm{~K}$. Everywhere else, the films responds like the prelayer.

As a stringent test of magnetometer performance, we have measured the local field gradient (Bean profile ${ }^{10}$ ) during vortex avalanches in the high field quantum $\operatorname{limit}^{11}$ in a single crystal of $\mathrm{YBa}_{2} \mathrm{Cu}_{3} \mathrm{O}_{7-\delta}$. We processed a $0.1 \mu \mathrm{m}$ InAs epilayer grown on GaAs into a six element Hall probe array with active areas $(8 \mu \mathrm{m} \times 8 \mu \mathrm{m})$ spaced $20 \mu \mathrm{m}$ apart using conventional photolithography and wet chemical etching in $\mathrm{HCl}: \mathrm{HNO}_{3}: \mathrm{H}_{2} \mathrm{O}(1: 1.5)$. Contacts were prepared using pure In dots annealed at $200{ }^{\circ} \mathrm{C}$ for $2 \mathrm{~h}$ under a flow of $5 \% \mathrm{H}_{2}$ and $95 \% \mathrm{~N}_{2}$. The magnetometer was affixed via a

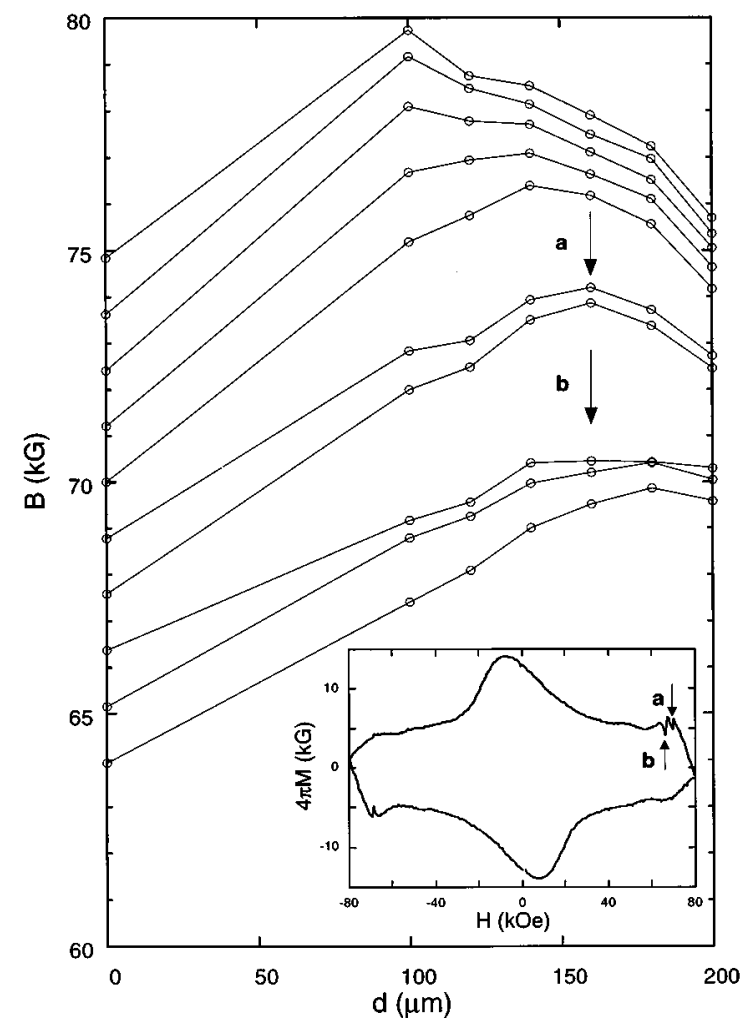

FIG. 5. Local internal field gradient using a six element InAs Hall probe array during vortex avalanches ( $\mathbf{a}$ and $\mathbf{b}$ ) at $T=0.35 \mathrm{~K}$ in a single crystal of $\mathrm{YBa}_{2} \mathrm{Cu}_{3} \mathrm{O}_{7-\delta}$. (Inset) Magnetization hysteresis loop. Data in the main part of the figure were taken on ramping the field down from $80 \mathrm{kOe}$. thin layer of grease to the regularly-shaped $(650 \mu \mathrm{m} \times 400$ $\mu \mathrm{m} \times 20 \mu \mathrm{m}$ thick) platelet along an axis of symmetry. Fourprobe resistance measurements were made using an ac lock-in technique in the frequency independent and ohmic limits.

We plot in Fig. 5 the internal magnetic field $B$ as a function of the distance $d$ from the edge of the crystal upon ramping the external field $H$ down from 75 to $64 \mathrm{kOe}$ at $T$ $=0.35 \mathrm{~K}$. The nonmonotonic variation of $B(d)$ reflects the hysteretic nature of the response. The magnetization jumps labeled $\mathbf{a}$ and $\mathbf{b}$ in the hysteresis loop shown in the inset correspond to changes of a thousand vortices under the gaussmeter. These avalanches show up clearly in the field profile, where the uneven spacing between curves near the center of the crystal contrasts with the approximately even spacing at the edge where $B=H$.

The resolution of the InAs elements is $<50 \mathrm{mG}$, at least 20 times better than comparable Bi probes, ${ }^{12}$ and is set here by the number of digits on the resistance bridge. For an 8 $\mu \mathrm{m} \times 8 \mu \mathrm{m}$ magnetometer, this corresponds to a sensitivity $\sim 0.1 \phi_{0}$, where $\phi_{0}$ is the fundamental unit of flux. The great sensitivity and temperature independence of InAs gaussmeters promise high-resolution studies down to the individual vortex level in both the quantum and classical regimes. ${ }^{13}$

The authors are grateful to H. Jaeger for illuminating discussions. The work at the University of Chicago was supported by the National Science Foundation (Contract No. DMR91-20000) through the Science and Technology Center for Superconductivity. EP acknowledges support from Argonne National Laboratory Department of Educational Programs. SPW acknowledges the support of the Natural Sciences and Engineering Research Council of Canada.

${ }^{1}$ T. Tamegai, L. Krusin-Elbaum, L. Civale, P. Santhanan, M. J. Brady, W. T. Masselink, F. Holtzberg, and C. Feild, Phys. Rev. B 45, 8201 (1992).

${ }^{2}$ S. P. Watkins, C. A. Tran, R. Ares, and G. Soerensen, Appl. Phys. Lett. 66, 882 (1995).

${ }^{3}$ N. F. Mott, The Metal-Insulator Transition (Taylor and Francis, London, 1974).

${ }^{4}$ J. E. Simpkins, Rev. Sci. Instrum. 39, 570 (1968).

${ }^{5}$ M. Konczykowski, F. Holtzberg, and P. Lejay, Semicond. Sci. Technol. 4, S331 (1991)

${ }^{6}$ Z. Koziol and J. J. M. Franse, IEEE Trans. Magn. 30, 1172 (1994).

${ }^{7}$ M. A. Paalanen, D. C. Tsui, and A. C. Gossard, Phys. Rev. B 25, 5566 (1982).

${ }^{8}$ See, for example, Y. Abulafia, A. Shaulov, Y. Wolfus, R. Prozorov, L. Burlachkov, Y. Yeshurun, D. Majer, E. Zeldov, and V. M. Vinokur, Phys. Rev. Lett. 77, 1596 (1996).

${ }^{9}$ H. H. Wieder, Appl. Phys. Lett. 25, 206 (1974).

${ }^{10}$ C. P. Bean, Phys. Rev. Lett. 8, 250 (1962).

${ }^{11}$ G. T. Seidler, C. S. Carillo, T. F. Rosenbaum, U. Welp, G. W. Crabtree, and V. M. Vinokur, Phys. Rev. Lett. 70, 2814 (1993); R. J. Zieve, T. F. Rosenbaum, H. M. Jaeger, G. T. Seidler, G. W. Crabtree, and U. Welp, Phys. Rev. B 53, 11849 (1996).

${ }^{12}$ K. M. Beauchamp, L. Radzihovsky, E. Shung, T. F. Rosenbaum, U. Welp, and G. W. Crabtree, Phys. Rev. B 52, 13025 (1995).

${ }^{13}$ K. M. Beauchamp, T. F. Rosenbaum, U. Welp, G. W. Crabtree, and V. M. Vinokur, Phys. Rev. Lett. 75, 3942 (1995); L. Radzihovsky ibid., 74, 4919 (1995). 